\title{
Transport Systems Conferring Pathogenicity to Escherichia coli
}

\author{
Fengyi Tang and Milton H. Saier $\mathrm{Jr}^{*}$ \\ Department of Molecular Biology, Division of Biological Sciences, University of California, USA \\ *Corresponding Author: Milton H. Saier Jr, Department of Molecular Biology, Division of Biological Sciences, University of California, USA, Tel: (858) 534-4084; Fax: \\ (858) 534-7108; E-mail: msaier@ucsd.edu
}

Rec Date: Aug 02, 2014, Acc date: Aug 29, 2014, Pub date: Aug 31, 2014

Copyright: (c) 2014 Saier Jr. This is an open-access article distributed under the terms of the Creative Commons Attribution License, which permits unrestricted use, distribution, and reproduction in any medium, provided the original author and source are credited.

\begin{abstract}
Escherichia coli K12 are currently the best-understood organism on Earth; a larger fraction of its genes have been functionally characterized than for any other organisms. However, organisms given this species designation are extremely diverse, genetically and phenotypically. E. coli proves to be a valuable model system for understanding bacterial physiology, metabolism, genetics and pathogenesis.

Why has E. coli been so much more extensively studied than any other bacterium on Earth? It all began with the German pediatrician and bacteriologist, Theodor Escherich, who was dismayed to find that so many babies were dying of diarrhea diseases. In fact, we now know that $\mathrm{E}$. coli is one of the top causes of infant mortality, adult diarrhea and urinary tract infections worldwide. It was because of the organism's broad host specificity and ability to cause many different diseases in large numbers of animals, including humans, that it was first selected as an important bacterium for study.

More recently, it has been found that certain strains of $\mathrm{E}$. coli are probiotic, having beneficial effects on host animals, counteracting the detrimental consequences of their pathogenic counterparts. Genetic analyses have revealed that the tremendous phenotypic diversity of the hundreds of currently recognized E. coli strains resulted from the frequent occurrence of horizontal gene transfer during the evolutionary divergence of the Escherichia genus. In fact, the pan genome of E. coli far exceeds the core genome, by up to 20 -fold or more. Thus E. coli should not be thought of as an entity or species; it represents an entire spectrum of related organisms sharing only the core housekeeping genes.
\end{abstract}

\section{Introduction}

Prominent types of E. coli pathogens have been identified based on varying mechanisms of infection. There are five types of diarrhoeagenic strains that pertain specifically to the alimentary tract: enteropathogenic (EPEC), enterohaemorrhagic (EHEC), enterotoxic (ETEC), enteroinvasive (EIEC), and enteroaggregative (EAEC). Additionally, uropathogenic E. coli (UPEC) strains have the ability to escape through the circulatory system and infect cells in the urinary tract, thereby causing urinary tract infections (up to $80 \%$ of UTI's worldwide are caused by E. coli strains) and pyelonephritis (inflammation of the kidneys) [1-4]. The mechanistic progression of E. coli virulence can be summarized as follows: (1) adhesion to host cell, mediated by cell-surface bacterial adhesins, (2) secretion of virulence factors into the target cell through various protein secretion systems, (3) hijacking of host cell signaling mechanisms using bacterially produced proteinaceous agents, (4) colonization of the host extracellular and/or intracellular environments, and (5) utilization of available nutrients for growth [5].

Transport proteins contribute significantly to each of these processes. Outer membrane secretion systems export adhesins, allowing $E$. coli adherence to target cells. In fact, in a recent study, we were able to distinguish several adhesins and fimbrial proteins that characterize each of the various pathogenic E. coli strains from the others and from K12 with respect to host cell targeting [6]. Secretion of virulence factors can be accomplished through various types of transenvelope protein secretion systems (Types I-IV; Type VI).
Different types of pathovars utilize distinct sets of protein secretion systems, including Type VI secretion systems that facilitate competition with other microbes. Iron acquisition is essential for the maintenance of E. coli metabolism, especially during colonization stages of infection. We have identified pathogen-specific inner membrane ABC-type iron shuttling systems as well as their cofunctioning outer membrane iron-siderophore receptors. Lastly, we examined carbon utilization in intracellular and extracellular pathogens and discovered striking differences [6]. We will discuss each of these findings in the subsequent sections of this review.

\section{Pore-Forming Toxins}

Pore-forming toxins are virulence factors that cause animal cell death, often due to leakage of essential cellular constituents. These toxins cause loss of cellular ions, proteins, and other molecules, but they sometimes also allow entry of specific toxins that affect host cell signaling. Pathogenic E. coli can thus utilize some of these toxins to hijack host cell machineries (e.g., cytoskeletal manipulation), further facilitating colonization of the host. These toxic pore-formers may also promote formation of biofilms (extracellular pathogens) or the generation of intracellular bacterial communities. We were able to identify Type III secretion system (T3SS) pore-forming proteins (IIITCP) in enteropathogenic strains that serve to provide translocation functions for E. coli effector proteins that inhibit host phagocytosis [4]. The EspB/D complex, the prototypical example of a IIITCP, was identified in two representative enteropathogenic strains although it was absent in K12 [6]. These proteins alter host cell 
cytoskeletal functions, particularly those pertaining to phagocytosis [7].

While we were able to confirm the presence of well-characterized pore-forming toxins in the various strains (e.g., shiga toxin, Stx, a heteromeric protein present in enterohemorrhagic strains that lead to disruption of host protein synthesis); previously unrecognized poreforming toxins were also identified in various pathovars [6]. For example, the clostridial cytotoxins (CCT) are tripartite proteins normally found in Gram-positive firmicutes, but homologues of CCT in E. coli strain ABU 83972, a uropathogenic E. coli pathovar, and O157:H7, an enterohemorrhagic pathovar, were identified. They form channels and inactivate Rho-type GTPases in the target cells, leading to cytoskeletal manipulation [8]. The presence of such homologues in the O157:H7 strain was surprising since EHEC strains typically utilize mitochondrion-associated proteins to inhibit host cell control proteins (e.g., CDC42) for cytoskeletal manipulation purposes. Additional studies can be conducted to elucidate whether alternative mechanisms of cytoskeletal manipulation are present in EHEC strains.

Serratia-type pore-forming toxins (S-PFT) proved to be present in a wide variety of pathovars, namely ABU 83972 (UPEC), O157:H7 (EHEC), and CFT073 (UPEC). S-PFT toxins are pore-forming haemolysins that are often exported via two-partner secretion systems [9] as well as Type VI secretion systems. The identification of twopartner secretion system(s) as well as Type VI secretion systems in ABU 83972 and O157:H7 strains suggest the possibility of novel Serratia-type pore-forming haemolysins being present in multiple E. coli pathogens. Further studies will be needed to establish the distribution of such systems and their mechanisms of action in the hundreds of known E. coli pathogens.

\section{Protein Secretion Systems}

Protein secretion systems can be categorized into three types: (1) transenvelope systems, and those that export proteins only across (2) the inner or (3) the outer membrane. Transenvelope secretion systems serve as the main mechanism of translocating virulence factors directly from the bacterial cytoplasm into the host cell cytoplasm. Depending on their location (intracellular versus extracellular), a pathogen utilizes different types of transenvelope protein secretion systems to specifically target host cells for injection or release of virulence factors into the internal or external environment. Many of the extracellular pathogens export proteins to the bacterial cell surface where they assist in adhesion to target cells. Various pathovars require different adhesion mechanisms to facilitate injection and colonization of host cells. We will briefly explore the unique characteristics of these protein secretion systems in the various E. coli pathovars.

\section{Transenvelope protein secretion systems}

Transenvelope protein secretion systems include types I, III, IV and VI systems. The major components of such systems include: (1) one or more inner membrane components, (2) an energizing protein such as an ATPase, (3) an "adaptor" protein or protein complex that spans the periplasm, forming a channel between the inner membrane and the outer membrane constituents of the system, and (4) an outer membrane protein or protein complex that serves as an outlet for transenvelope secretion [9].

Type I secretion systems (T1SSs) differ from types III and IV in that Type I systems (TC\#3.A.1) each consists of an ATP-dependent inner membrane energizer (the ABC ATPase), the transmembrane channel- forming protein (often fused to the ATPase), an adaptor, erroneously called a "membrane fusion protein" (MFP; TC\#8.A.1), and "an outer membrane factor" (OMF; TC\#1.B.17), allowing the exported virulence factor to be released directly into the extracellular environment without exposure to the periplasm (Figure 1) [10]. T1SSs are found mostly in intracellular pathogens that typically release their virulence factors while residing in the host's cytoplasm. We were able to identify T1SSs in ABU 83972, CFT073 and UMN026, all of which are UPEC strains that can operate intracellularly in the host cell to form intracellular bacterial communities. Interestingly, we found that O157:H7, an extracellular EHEC pathovar, also possesses essential components of a T1SS system. Further studies will be required to establish its function in EHEC pathogenicity.

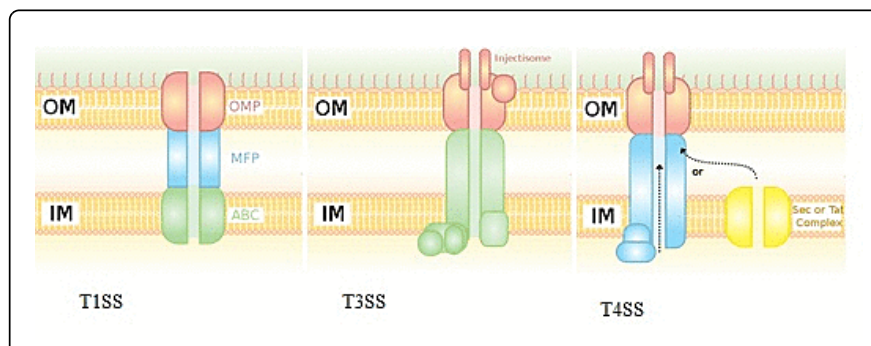

Figure 1: Illustration of three types of transenvelope protein export systems present in various E. coli pathogens.

Types III and IV secretion systems, consist of an injectisome (T3SS) or an injectisome-like complex (T4SS) that allows the pathogen to specifically target host cells for injection (Figure 1). These systems are primarily useful to extracellular pathogens that inject their virulence factors directly into the cytoplasm of the target cell. T3SSs and T4SSs are both multicomponent systems that differ primarily with respect to their substrates; T3SSs primarily transport effector proteins while T4SSs typically transport macromolecules including nucleoprotein complexes [11,12]. As expected, we were able to identify T3SSs for secretion of flagellar and virulence factors in extracellular pathogens 55989 (EAEC) and E24377A (ETEC). T4SSs were abundant in UMN026 (UPEC) and O157:H7 (EHEC).

Type VI secretion systems (T6SSs) were first identified in 2006 by John Mekalanos' research group studying V. cholerae and P. aeruginosa [13]. A T6SS has an inner membrane ATP-dependent energizer like T1SSs, but it also possesses a phage-like tail in its outer membrane (Figure 2). These systems serve a primary function in interbacterial competition, although they also may inject proteins into the host cell cytoplasm. The phage-tail consists of a needle-like complex and a dissociable cap homologous to the needle complex of lambda phage. Several studies suggest that certain bacterial strains acquired this mechanism via horizontal gene transfer and utilize the T6SS to target other bacterial cells for destruction [14]. Host cell virulence factors secreted through T6SSs include cell growth inhibitors and apoptosis promoting agents.

We identified sets of homologous essential components of the T6SS in pathovars 55989 (EAEC), APEC O1 (AIEC), E24377A (ETEC), UMN026 (UPEC) and O157:H7 (EHEC). T6SSs had previously been identified in strains 55989 and APEC O1 [15-17]. The homologous T6SSs identified in other strains may provide clues as to how these pathogenic strains compete for survival in the host's microbiome. T6SSs allow pathogenic E. coli strains to engage in T6SS-mediated attacks (and counter attacks) against other cells in its proximal 
environment [18], providing a mechanism that can facilitate their colonization success in the densely packed host environment where numerous facultative anaerobes compete for limited metabolic resources.

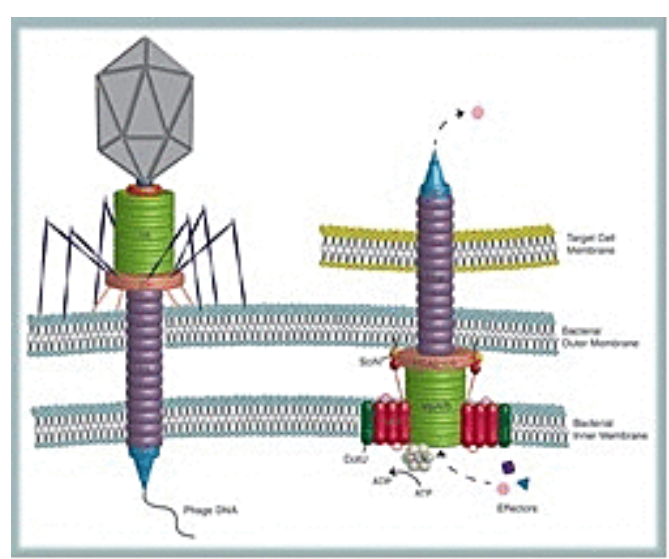

Figure 2: Schematic depiction of a type VI secretion system. ClpV is the phage-like tail and VgrG is the dissociable tail spike. See [15] and [16] for recent reviews.

\section{Outer membrane protein secretion systems}

Outer membrane protein secretion systems include Autotransporter-1 (AT-1), Autotransporter-2 (AT-2), and putative Autotransporter-3 (AT-3) (intimin and invasin) systems. While transenvelope protein secretion systems primarily assist in the secretion of virulence factors, outer membrane secretion systems often export factors that assist E. coli in host cell adhesion or proteolysis [19]. The human digestive tract replenishes old layers of villi and mucosal cells with new cell layers, and effective adhesion mechanisms are important for pathogens to successfully colonize the rapidly changing host cell environments. AT-1, AT-2 and AT-3 systems can also play essential roles in biofilm formation [20].

Among the strains examined in our study, AT-2 systems were found in APEC O1 (respiratory tract), UMN026 (urinary tract), and O157:H7 (colonic tract). AT-1 and AT-2 systems transport adhesins, proteases and toxins through outer membrane $\beta$-barrel pores. Intimins and invasins were identified in almost all strains except for 55989. Examples include the Tir (Adhesion/Effacing) pathway, which triggers cytoskeletal rearrangement in the host cells upon pathogen binding to beta-1 integrin receptors on target cells [21]. In addition, numerous fimbrial usher proteins and secretins were found in greater numbers in pathovars than in the nonpathogenic K12. The exact mechanisms by which they operate in adhesion and biofilm formation are in need of further exploration.

\section{Iron Acquisition}

Iron plays an important role in E. coli metabolism because of its participation in the electron transport chain and as a cofactor in numerous enzymes. Various cytochromes, iron-sulfur proteins and ferric oxidases that operate in the electron transport chain exhibit greatly reduced efficiency when iron is lacking in the in vitro environment. Our immune responses to bacterial infections include upregulating iron sequestration mechanisms in the digestive tract to decrease iron available to the pathogens [22]. Interestingly, we were able to identify both an abundance of iron-siderophore outer membrane receptors as well as their corresponding high-affinity inner membrane ABC-type uptake systems, found in increased numbers in pathogenic strains [6]. We speculate that such systems of iron acquisition allow pathogens to sustain colonization by effectively competing with host iron sequestration.

Examples abound. Ferric (Fe3+) catecholate receptors (TC\#1.B. 14.1.4), ferric yersiniabactin uptake receptors (1.B.14.7.2), ferrienterobactin receptors (1.B.14.1.3) and ferrichrome receptors (1.B. 14.1.15) were identified exclusively in UPEC strains (ABU 83972, UMN026 and CFT073). Corresponding high affinity ABC transporters $(\mathrm{Km}$ values $\leq 1 \mu \mathrm{M})$ were found either exclusively in these UPEC strains or in significantly greater numbers than in K12. In fact, when we graphed numbers of outer membrane receptors vs. numbers of inner membrane siderophore shuttling systems, we found a strong correlation $(\mathrm{R}=0.90)$ between the two variables, suggesting that the two probably coevolved or were co-acquired, and therefore serve as sequential agents of iron acquisition [6].

By contrast, low affinity secondary carriers (TC Subclass 2.A; Km values of $\sim 10-800 \mu \mathrm{M}$ ) were found to be ubiquitous and in equal numbers in strains of E. coli studied, including K12. We thus concluded that high affinity iron siderophore shuttling systems, including environment-specific iron-sideorphore receptors and corresponding ABC-uptake systems, serve virulence purposes and are likely to have co-evolved or have been co-acquired. Low affinity iron siderophore carriers, on the other hand, probably play a housekeeping role in all strains of $\mathrm{E}$. coli.

\begin{tabular}{|c|c|c|c|c|c|}
\hline Strain & $\begin{array}{l}\text { Genome } \\
\text { size (Mbp) }\end{array}$ & $\begin{array}{ll}\# & \text { of } \\
\text { Proteins }\end{array}$ & Host Location & $\begin{array}{l}\text { Pathological } \\
\text { condition }\end{array}$ & $\begin{array}{l}\text { Pathovar } \\
\text { Type }\end{array}$ \\
\hline 55989 & 5.15 & 4,759 & Extracellular & Diarrhoea & EAEC \\
\hline $\begin{array}{l}\text { ABU } \\
83972\end{array}$ & 5.13 & 4,796 & Intracellular & Bacteremia & UPEC \\
\hline APEC 01 & 5.50 & 4,853 & Intracellular & $\begin{array}{l}\text { Respiratory } \\
\text { infection } \\
\text { (avians) }\end{array}$ & AIEC \\
\hline CFT073 & 5.23 & 5,369 & Intracellular & $\begin{array}{l}\text { Urinary tract } \\
\text { infection } \quad \& \\
\text { pyelonephritis }\end{array}$ & UPEC \\
\hline E24377A & 5.25 & 4,991 & Extracellular & Enterotoxicity & ETEC \\
\hline UMN026 & 5.36 & 5,014 & $\begin{array}{l}\text { Extra/Intra } \\
\text {-cellular }\end{array}$ & $\begin{array}{l}\text { Urinary } \\
\text { infection }\end{array}$ & UPEC \\
\hline O157:H7 & 5.70 & 5,477 & Extracellular & $\begin{array}{l}\text { Haemorrhagic } \\
\text { colitis }\end{array}$ & EHEC \\
\hline $\begin{array}{l}\text { K12 } \\
\text { MG1655 }\end{array}$ & 4.64 & 4,290 & Extracellular & None & $\begin{array}{l}\text { Non- } \\
\text { virulent }\end{array}$ \\
\hline
\end{tabular}

Table 1: Seven E. coli pathovars, the non-virulent E. coli K12 strain, and their basic traits

\section{Intracellular Vs. Extracellular Nutrient Transport}

E. coli pathogens can be grouped into two general categories: extracellular and intracellular (Table 1). Extracellular E. coli (EAEC, ETEC, EHEC, and certain UPEC strains such as UMN026) typically 
utilize attaching/effacing mechanisms to stay outside of host cells. During biofilm formation, these strains utilize nutrients present external to the host cell. Intracellular pathogens, on the other hand, colonize host cells and form intracellular bacterial communities. A striking pattern was revealed by our study suggesting that intracellular and extracellular E. coli pathovars possess distinctive sets of nutrient porters, distinguishable based on the substrates transported (Table 2) [6].

\begin{tabular}{|l|l|l|l|}
\hline $\begin{array}{l}\text { Transporter } \\
\text { (TC\#) }\end{array}$ & Substrate & Intracellular & Extracellular \\
\hline 2.A.56.1.6 & Sialic acid & $\mathrm{x}$ & \\
\hline 2.A.80.1.1 & Tricarboxylate & $\mathrm{x}$ & \\
\hline 3.A.1.2.1 & Ribose & $\mathrm{x}$ & \\
\hline 3.A.1.5.12 & Rhamnose oligosaccharide & & $\mathrm{x}$ \\
\hline 4.A.1.1.3 & Maltose & $\mathrm{x}$ & \\
\hline 4.A.2.1.3 & 2-O-a-Mannosyl-D glycerate & & $\mathrm{x}$ \\
\hline 4.A.2.1.6 & Mannose & & $\mathrm{x}$ \\
\hline 4.A.2.1.9 & Keto sugar & $\mathrm{x}$ & \\
\hline 4.A.4.1.2 & Sugar alcohol (glucitol) & & $\mathrm{x}$ \\
\hline 4.A.5.1.3 & Sugar alcohol (galactitol) & & $\mathrm{x}$ \\
\hline 4.B.1.1.4 & Coenzyme precursor (thiamin) & $\mathrm{x}$ & \\
\hline
\end{tabular}

Table 2: A few representative metabolite uptake transporters characteristic of intracellular vs. extracellular pathogens. Adapted from Tang \& Saier, 2014

For example, rhamnose oligosaccharide porters (3.A.1.5.12), $\alpha$ glucoside-specific IICB permeases of the phosphotransferase system (PTS) (4.A.1.1.10), and 2-O- $\alpha$-mannosyl-D-glycerate PTS porters (4.A. 2.1.3) were found exclusively in extracellular pathogens but absent in intracellular pathogens. In general, oligosaccharides and disaccharides are present in the extracellular environment but absent inside the host cell cytoplasm. Extracellular pathogens therefore possess transporters that allow the bacteria to take advantage of these nutrients.

By contrast, transporters that target monosaccharides (e.g., ATPdependent ribose porters (3.A.1.2.1), D-tagatose group translocators (4.A.2.1.9)) and TCA cycle intermediate uptake systems (e.g., Citrate:H+ symporters (2.A.1.6.1) and fumarate-malate antiporters (2.A.13.1.2)) were found almost exclusively in intracellular pathogens. Since intracellular pathogens initiate colonization inside the cytoplasm of the host cell, these strains likely utilize these transporters to take advantage of metabolites found almost exclusively in intracellular environments. The presence of these distinct nutrient transporters points to the likelihood of frequent gain or loss of carbon sourcespecific transport systems, probably via horizontal gene transfer, in response to their specific biological niches. Intracellularity may be a relatively recent phenomenon $[23,24]$. The clear distinctions drawn in our studies between the two types of pathovars [6] could be of diagnostic value in future clinical studies.

\section{Concluding Remarks}

Escherichia coli have been a model pathogen for scientific study due in large measure to its diverse pan-genome and wide spectrum of pathogenesis. Upon examining the transport systems of seven wellcharacterized E. coli pathogens causing urinary tract infections, gastroenteritis, pyelonephritis and haemorrhagic colitis, we were able to observe significant patterns of transport protein distributions related to pathogenesis. Each pathogen possesses a unique set of protein secretion systems for export to the cell surface or for injecting effector proteins into host cells. We were able to identify protein constituents of homologous Type VI secretion systems in pathovars previously not known to possess them. Pathogens have increased numbers of iron siderophore receptors and $\mathrm{ABC}$ iron uptake transporters, although the numbers and types of low-affinity secondary iron carriers were uniform in all strains. The presence of outer membrane iron complex receptors and high-affinity $\mathrm{ABC}$ iron uptake systems correlated, suggesting co-evolution or co-acquisition. Furthermore, each pathovar encodes a different set of pore-forming toxins and virulence-related outer membrane proteins lacking in E. coli K12. Lastly, intracellular pathogens proved to have a characteristically distinctive set of nutrient uptake porters, different from those of extracellular pathogens. Such distinctions can be useful in evolutionary studies of gene transfer as well as for diagnostic or epidemiological purposes for identifying pathogen types based on their proximal biological habitats.

\section{References}

1. Williams EJ, Embleton ND, Bythell M, Ward Platt MP, Berrington JE (2013) The changing profile of infant mortality from bacterial, viral and fungal infection over two decades. Acta Paediatr 102: 999-1004.

2. Zhang L, Foxman B, Manning SD, Tallman P, Marrs CF (2000) Molecular epidemiologic approaches to urinary tract infection gene discovery in uropathogenic Escherichia coli. Infect Immun 68: 2009-2015.

3. Huebner C, Ding Y, Petermann I, Knapp C, Ferguson LR (2011) The probiotic Escherichia coli nissle 1917 reduces pathogen invasion and modulates cytokine expression in caco-2 cells infected with crohn's disease-associated E. coli lf82. Appl Environ Microbiol 77: 2541-2544.

4. Croxen MA, Finlay BB (2010) Molecular mechanisms of Escherichia coli pathogenicity. Nat Rev Microbiol 8: 26-38.

5. Bhavsar AP, Guttman JA, Finlay BB (2007) Manipulation of host-cell pathways by bacterial pathogens. Nature 449: 827-834.

6. Tang F, Saier MH Jr2 (2014) Transport proteins promoting Escherichia coli pathogenesis. Microb Pathog 71-72: 41-55.

7. Iizumi Y, Sagara H, Kabe Y, Azuma M, Kume K, Ogawa M, et al. (2007) The enteropathogenic E. coli effector espb facilitates microvillus effacing and antiphagocytosis by inhibiting myosin function. Cell Host Microbe 2: 383-392.

8. Oswald E, Sugai M, Labigne A, Wu HC, Fiorentini C, Boquet $\mathrm{P}$, et al. (1994) Cytotoxic necrotizing factor type 2 produced by virulent Escherichia coli modifies the small gtp-binding proteins rho involved in assembly of actin stress fibers. Proc Natl Acad Sci U S A 91: 3814-3818.

9. Hertle R (2005) The family of serratia type pore forming toxins. Curr Protein Pept Sci 6: 313-325.

10. Yen MR, Peabody CR, Partovi SM, Zhai Y, Tseng YH, et al. (2002) Protein-translocating outer membrane porins of Gram-negative bacteria. Biochim Biophys Acta 1562: 6-31.

11. Alvarez-Martinez CE, Christie PJ (2009) Biological diversity of prokaryotic type IV secretion systems. Microbiol Mol Biol Rev 73: 775-808.

12. Diepold A, Wiesand U, Cornelis GR (2011) The assembly of the export apparatus $(Y s c R, S, T, U, V)$ of the Yersinia type III secretion apparatus 
Citation: Tang F, Saier Jr (2014) Transport Systems Conferring Pathogenicity to Escherichia coli. J Med Microb Diagn 3: 159. doi: 10.4172/2161-0703.1000159

Page 5 of 5

occurs independently of other structural components and involves the formation of an YscV oligomer. Mol Microbiol 82: 502-514.

13. Pukatzki S, Ma AT, Revel AT, Sturtevant D, Mekalanos JJ (2007) Type vi secretion system translocates a phage tail spike-like protein into target cells where it cross-links actin. Proc Natl Acad Sci U S A 104: 15508-15513.

14. Pukatzki S, Kessin RH, Mekalanos JJ (2002) The human pathogen pseudomonas aeruginosa utilizes conserved virulence pathways to infect the social amoeba dictyostelium discoideum. Proc Natl Acad Sci U S A 99: 3159-3164.

15. Russell AB, Peterson SB, Mougous JD (2014) Type VI secretion system effectors: poisons with a purpose. Nat Rev Microbiol 12: 137-148.

16. Ho BT, Dong TG, Mekalanos JJ (2014) A view to a kill: The bacterial type vi secretion system. Cell Host Microbe 15: 9-21.

17. de Pace F, Boldrin de Paiva J, Nakazato G, Lancellotti M, Sircili MP, et al. (2011) Characterization of IcmF of the type VI secretion system in an avian pathogenic Escherichia coli (APEC) strain. Microbiology 157: 2954-2962.

18. Basler M, Ho BT, Mekalanos JJ (2013) Tit-for-tat: Type vi secretion system counterattack during bacterial cell-cell interactions. Cell 152: 884-894.
19. Tükel C, Akçelik M, de Jong MF, Simsek O, Tsolis RM, et al. (2007) MarT activates expression of the MisL autotransporter protein of Salmonella enterica serotype Typhimurium. J Bacteriol 189: 3922-3926.

20. Arenas J, Cano S, Nijland R, van Dongen V, Rutten L, et al. (2014) The meningococcal autotransporter AutA is implicated in autoaggregation and biofilm formation. Environ Microbiol.

21. Schuller S, Chong Y, Lewin J, Kenny B, Frankel G, Phillips AD et al. (2007) Tir phosphorylation and nck/n-wasp recruitment by enteropathogenic and enterohaemorrhagic Escherichia coli during ex vivo colonization of human intestinal mucosa is different to cell culture models. Cell Microbiol 9: 1352-1364.

22. Cherayil BJ (2011) The role of iron in the immune response to bacterial infection. Immunol Res 50: 1-9.

23. Qazi SN, Harrison SE, Self T, Williams P, Hill PJ (2004) Real-time monitoring of intracellular Staphylococcus aureus replication. J Bacteriol 186: 1065-1077.

24. Guan SH, Gris C, Cruveiller S, Pouzet C, Tasse L, et al. (2013) Experimental evolution of nodule intracellular infection in legume symbionts. ISME J 7: 1367-1377. 